\title{
Global Health Crisis, Global Health Response: How Global Health Experiences Prepared North American Physicians for the COVID-19 Pandemic
}

\author{
Alexandra L. Coria, MD ${ }^{7,2} \mathbb{B}_{0}$, Tracy L. Rabin, MD, SMY3, Amy R.L. Rule, MD, MPH ${ }^{4,5}$, \\ Heather Haq, MD, $M H S^{6,7}$, James C. Hudspeth, $\mathrm{MD}^{8}$, Leah Ratner, MD, MS, DTM\& $H^{9,10,11}$, \\ and Ingrid Walker-Descartes, MD, MPH, MBA ${ }^{2,12}$
}

\begin{abstract}
'Global Health Institute and Division of Pediatric Hospital Medicine, Maimonides Medical Center, Brooklyn, NY, USA; ${ }^{2}$ State University of New York Downstate College of Medicine, Brooklyn, NY, USA; ${ }^{3}$ Office of Global Health \& Yale Primary Care Internal Medicine Residency Program,

Department of Internal Medicine, Yale School of Medicine, New Haven, CT, USA; ${ }^{4}$ University of Cincinnati College of Medicine, Cincinnati, OH, USA; ${ }^{5}$ Perinatal Institute and Division of Hospital Medicine, Department of Pediatrics, Cincinnati Children's Hospital Medical Center, Cincinnati, OH, USA; ${ }^{6}$ Baylor College of Medicine International Pediatric AIDS Initiative (BIPAI) at Texas Children's Hospital, Houston, TX, USA; ${ }^{7}$ Department of Pediatrics, Baylor College of Medicine, Houston, TX, USA; ${ }^{8}$ Department of Medicine, Section of General Internal Medicine, Boston University, Boston, MA, USA; ${ }^{9}$ Harvard Medical School, Boston, MA, USA; ${ }^{10}$ Division of Respiratory Diseases, Boston Children's Hospital, Boston, MA, USA; ${ }^{11}$ General Internal Medicine and Primary Care, Brigham and Women's Hospital, Boston, MA, USA; ${ }^{2}$ Division of Medical Education, Maimonides Children's Hospital, Brooklyn, NY, USA.
\end{abstract}

The COVID-19 pandemic plunged hospital systems into resource-deprived conditions unprecedented since the 1918 flu pandemic. It brought forward concerns around ethical management of scarcity, racism and distributive justice, cross-disciplinary collaboration, provider wellness, and other difficult themes. We, a group of medical educators and global health educators and clinicians, use the education literature to argue that experience gained through global health activities has greatly contributed to the effectiveness of the COVID-19 pandemic response in North American institutions. Support for global health educational activities is a valuable component of medical training, as they build skills and perspectives that are critical to responding to a pandemic or other health system cataclysm. We frame our argument as consideration of three questions that required rapid, effective responses in our home institutions during the pandemic: How can our health system function with new limitations on essential resources? How do we work at high intensity and volume, on a new disease, within new and evolving systems, while still providing high-quality, patient-centered care? And, how do we help personnel manage an unprecedented level of morbidity and mortality, disproportionately affecting the poor and marginalized, including moral difficulties of perceived care rationing?

KEY WORDS: global health education; global health; medical education; COVID-19.

$\mathrm{J}$ Gen Intern Med 37(1):217-21

DOI: $10.1007 / \mathrm{s} 11606-021-07120-\mathrm{w}$

(c) Society of General Internal Medicine 2021

Prior Presentations None.

Received March 26, 2021

Accepted August 25, 2021

Published online September 24, 2021

\section{INTRODUCTION}

The COVID-19 pandemic has profoundly affected health institutions, plunging inpatient services and whole hospital systems - indeed, whole countries - into resource-limited conditions unprecedented since the 1918 influenza pandemic ${ }^{1}$. Furthermore, communities already affected by racism, poverty, and other forms of structural violence have disproportionately borne the morbidity and mortality burden of COVID$19^{2,3}$. As US-based global health professionals and medical educators, we know that resource and outcome disparities are often directly traceable to colonialism and other racist structures, and we are committed to promoting care that is decolonialist, anti-racist, and based on bidirectional exchange of knowledge, skills, and resources.

We see broad applicability of our work in global health-a field fundamentally about improving health equity ${ }^{4}$-in the pandemic response. We found that as acuity and volume surged on inpatient services in our home institutions, the challenges to providing high-quality, equitable care were numerous, but not unfamiliar. In addressing these challenges, we often called on skills learned while working with partners who practice primarily in resource-constrained settings.

Even as we strive to support our partners as they face severe resource shortages themselves, we see an opportunity to highlight for our colleagues working in high-resource environments how global health experiences (GHEs) have been essential to the pandemic response in our home institutions (Table 1).

We reviewed the literature, identifying areas where clinical trainees from high-income settings report how GHEs influence their knowledge, skills, and attitudes ${ }^{5-9}$. We then identified three questions that required rapid responses in our institutions during the pandemic, demonstrating here how GHEs 
Table 1 Examples of Benefits of GHEs Drawn from the Medical Education Literature that Were Directly Applied During the COVID-19 Pandemic

\begin{tabular}{|c|c|}
\hline Benefit of GHE & Application during COVID-19 pandemic \\
\hline Resourcefulness, innovation & $\begin{array}{l}\text { - Reprocessing single-use equipment } \\
\text { - Quickly learning to operate new ventilators or monitors } \\
\text { - Taking creative advantage of untraditional resources: hotel rooms for isolation, construction } \\
\text { supply chains for PPE }\end{array}$ \\
\hline $\begin{array}{l}\text { Increased physical exam skills, less reliance on } \\
\text { diagnostics and imaging }\end{array}$ & - Assessing respiratory status without timely labs/imaging due to volume-related delays \\
\hline $\begin{array}{l}\text { Greater awareness of resource cost, systems-based } \\
\text { practice }\end{array}$ & $\begin{array}{l}\text { - Critically assessing, with interdisciplinary team members, benefit vs. risk of intubation on an } \\
\text { individual basis, in order to manage ventilator allocation, balancing patient goals of care and } \\
\text { public health needs }\end{array}$ \\
\hline $\begin{array}{l}\text { Role flexibility, improved interdisciplinary } \\
\text { communication }\end{array}$ & $\begin{array}{l}\text { Physicians doing tasks typically performed by others but within their purview/skills, like } \\
\text { adjusting IV drips, proning, changing soiled sheets }\end{array}$ \\
\hline Ethical allocation of scarce resources & - Efficiently formulating equitable policies around ICU bed allocation, vaccine distribution \\
\hline $\begin{array}{l}\text { Help-seeking when practicing out of scope of } \\
\text { professional competency }\end{array}$ & $\begin{array}{l}\text { - Pediatricians, surgeons, non-intensivists seeking out resources and teachers, to practice safely } \\
\text { and ethically while treating patients not normally in their purview }\end{array}$ \\
\hline Learning new disease and epidemiology & - Clinicians on COVID units constantly integrating emerging information about the disease \\
\hline \multirow[t]{2}{*}{$\begin{array}{l}\text { Flexibility to operate within different healthcare } \\
\text { systems }\end{array}$} & $\begin{array}{l}\text { - Pediatricians designing new systems to access adult medicine resources when treating COVID } \\
\text { patients }\end{array}$ \\
\hline & $\begin{array}{l}\text { - New team structures to accommodate non-IM specialists treating adults } \\
\text { - Critical care consultants co-managing critical patients on the floor }\end{array}$ \\
\hline Cultural humility & $\begin{array}{l}\text { - Accommodating culture-driven end-of-life requests when family not present } \\
\text { - Listening and adapting to different practice habits across disciplines }\end{array}$ \\
\hline Impro & - Internal medicine specialists getting non-IM specialists up to speed quickly \\
\hline $\begin{array}{l}\text { Development of coping mechanisms/focusing on } \\
\text { positive outcomes }\end{array}$ & $\begin{array}{l}\text { - Using and helping others develop coping mechanisms to continue to achieve what good } \\
\text { outcomes were possible, despite high mortality and the frustration of the unavailability of } \\
\text { "normal" resources }\end{array}$ \\
\hline & - Debriefing teams working on COVID units \\
\hline Incre & $\begin{array}{l}\text { - Providers under stress, emotionally supporting both new colleagues they do not know well and } \\
\text { patients who had little family support. }\end{array}$ \\
\hline \multirow[t]{2}{*}{$\begin{array}{l}\text { Increased awareness of social determinants of } \\
\text { health }\end{array}$} & $\begin{array}{l}\text { - Counseling families about social distancing in a culturally sensitive way, taking into account } \\
\text { structural contributors to disease risk. }\end{array}$ \\
\hline & $\begin{array}{l}\text { - Advocating for resources to decrease disease risk among vulnerable populations } \\
\text { "White Coats for Black Lives" movement emphasizing link between racism and public } 1\end{array}$ \\
\hline
\end{tabular}

prepared individuals to help institutions in high-income settings effectively respond to them.

\section{Q1: How can our health system function with new limitations on essential resources?}

GHEs often place participants in settings that are higher acuity and more resource-limited than their home sites, and at the very least require participants to learn new ways of accessing familiar resources (e.g., access to different formularies). Learning from local mentors, GHE participants report boosted clinical resourcefulness and problem-solving ${ }^{6,10,11}$ as well as improved physical exam and procedural skills, with less reliance on testing and imaging 5 . During the pandemic, these attributes have been important when dealing with shortages in lab and imaging capacity, or the need to use available resources in new ways (e.g., reprocessing single-use equipment or repurposing transport ventilators for inpatient use). This resourcefulness extends to creatively mobilizing networks, like sourcing personal protective equipment from construction companies.

North American GHE participants also report increased sensitivity to resource cost to both the patient and the health system $^{12}$. This awareness allows physicians to better consider the needs of the hospital, health system, and community when acting as stewards of scarce resources. The ethics of equitably distributing scarce resources is a cornerstone of global health learning $6,10,13,14$.

A final element of operating with limited resources is being asked to practice outside one's scope of training ${ }^{6,13}$, often due to personnel shortages, and learning to proactively seek help. During the pandemic, individuals - including some of this paper's authors - have been purposely mobilized outside their scope of training: pediatricians caring for adults, surgeons on medical services, etc., requiring critical examination of our own abilities and limitations, and active, frequent help-seeking. GHEs, where this situation is common, help participants learn to navigate the tension between wanting to help and knowing when their unpracticed intervention will do more harm than good. Awareness of one's own limitations and when to seek help can manifest as active engagement with multidisciplinary teams, seeking out just-in-time teaching resources, or looking for someone more qualified via telehealth or other creative consultation and support structures, all of which were employed during the pandemic.

\section{Q2: How do we work at high intensity and volume, on a new disease, within rapidly evolving systems, while still providing high-quality, patient-centered care?}

As global health work often includes travel to settings with unfamiliar epidemiology, GHEs require self-directed learning 
and humbly listening to local medical experts ${ }^{8}$ to rapidly increase medical knowledge. GHEs also contribute to honing educational skills, including flexibly delivering on-the-spot teaching to trainees in unfamiliar clinical situations ${ }^{12}$. During the pandemic, physicians have learned disease information from colleagues of all positions and academic ranks, all over the world, and taught it to other, equally diverse teams, often under stress and time pressure.

GHEs also require that trainees and clinicians function in different social and clinical cultures ${ }^{8}$. Clinical specialties develop their own habits and dogmas, and during the pandemic, providers have been "deployed" into new roles, requiring cultural humility to function within interdisciplinary teams. For example, internists supervising pediatric residents deployed to their service may encounter different assumptions about intern autonomy, nursing communication, or family involvement. They need flexible attitudes about how to approach care, mindsets that are as much cultural as pragmatic.

Cultural humility was of further importance as healthcare workers relocated to bolster hard-hit workforces. Relief workers entered new hospitals with different professional cultures, not to mention patient populations with different social demographics from those they typically served.

The importance of adaptability also extends to the systems themselves. During GHEs, providers flex to function in health systems that have different strengths and challenges ${ }^{12}$. As new operational structures have been trialed and modified rapidly during the pandemic, versatility and creativity in systemsbased practice has been critical.

As new systems and policies have emerged, so has information about inequitable access to resources. Advocacy has been a prominent feature of the pandemic response-for example, for adequate PPE, vaccine equity, and patient needs while their loved ones and natural advocates cannot be at their bedsides. Effective advocacy is a core value of global health practice, and requires an understanding of the social determinants of health, a benefit of GHEs ${ }^{6,8}$, as well as partnership with disenfranchised communities.

For example, early in the pandemic, many American healthcare workers participated in demonstrations in opposition to race-based violence, despite understanding the risks of group gatherings at that time. For many, this decision acknowledged that racism and implicit bias are direct contributors to the disproportionate impact of the pandemic on people of color. For many global health professionals, spreading this message and devising harm reduction strategies (e.g., passing out masks at protests) are key elements of a patient-centered, holistic pandemic response $\mathrm{e}^{15,16}$.

Q3: How do we help personnel manage an unprecedented level of morbidity and mortality, disproportionately affecting the poor and marginalized, including moral difficulties of perceived care rationing?
The literature demonstrates that GHE participants from high-resource settings benefit from debriefing and mental health resources after re-entry ${ }^{6,17,18}$. Consistently, high mortality and its systemic contributors are topics of reflection and learning in these sessions ${ }^{7,9,11}$. During the pandemic, global health practitioners have recognized a similar need for timely debriefing. Specific tools have been developed to help the non-mental health professional lead debriefings after GHEs and global health simulations ${ }^{14,19,20}$, also relevant now.

Maintaining resilience and provider wellness in the face of high mortality is another benefit of GHEs ${ }^{7}$. Early in the pandemic, with limited and rapidly changing information about a new disease, acceptance of uncertainty was key. Focusing on factors that can be changed is a common coping technique among practitioners who deal with regular practicerelated trauma; an intensivist colleague from a resourcedeprived hospital in Uganda explained, "If our ICU has a $40 \%$ mortality rate...60\% of the patients survive, and those are the ones on [whom] we can make a profound impact." Similarly, one GHE participant wrote that "relishing the clinical win" was important to resiliency in resource-denied settings ${ }^{7,11}$. These lessons and strategies have been invaluable as the trauma of a disaster response and prolonged societal recovery has taken a mental health toll on health workers everywhere.

Finally, trainee reflections often credit GHEs for increasing empathy and humanism ${ }^{5}$, a critical aspect of patient and colleague interactions during these morally distressing times.

\section{CONCLUSION}

Even more than captured here, we have found perspectives shaped by GHEs to be immensely useful during the COVID19 pandemic. GHEs prepare practitioners to effectively respond to unexpected, acute, or difficult situations, particularly those that result in significant resource scarcity and exacerbate structural violence, leading to disproportionate effects on immigrants, the poor and communities of color.

We acknowledge that many - though not all—of the competencies addressed here can be supported through educational experiences that may not be categorized as GHEs. (For example, a rotation in a public health department or a workshop on cost-conscious medical decision-making.) However, we argue that those competencies are nonetheless supported in a unique way in GHEs. Many lecture topics are reinforced and made real with practical experience: for example, trying to make ethical medical decisions in the face of severe resource scarcity, or opting to seek help when asked to operate outside one's qualified scope of practice. Even skills like teaching, which are pervasive in medicine, acquire a different emphasis when the teacher is in a new cultural context, or under time or resource pressure, as one frequently is during GHEs, and many were during the pandemic. Similarly, humility, 
flexibility, and resilience are attitudes that are emphasized throughout medical training, but in very resource-challenged settings or situations, they become essential skills.

Further, the global health paradigm emphasizes that health disparities everywhere share similar drivers ${ }^{21}$. Many highresource institutions now offer local GHEs, acknowledging that one need not go far to find systemic drivers of health inequity. In these rotations, learners examine root causes of health inequity and resource shortages in their home communities and see up close disparities that exist even between blocks of the same city. They learn implementation science, advocacy, cultural humility, ethics of working with limited resources, and other fundamental global health competencies $^{22}$.

We assert that had we not engaged in GHEs through our training and careers, we would not have been as prepared to address the COVID-19 pandemic. Many of the authors took on leadership roles in our institutions' COVID-19 response, an unanticipated but logical extension of our global health training. In an increasingly globalized world, it is likely that we will face more situations, as a society, that require global health competencies. We argue, therefore, that GHEs are an indispensable element of medical education.

Additionally, while we have focused here on the value of GHEs in North American institutions recently hit hard by the pandemic, global health professionals also use their knowledge and skills to support the needs of partners in chronically resource-deprived settings - the very partners who have taught us valuable lessons about resourcefulness, ethics, distributive justice, resiliency, and other themes explored above. Examples of ongoing work include advocacy for equity in global vaccine distribution and expanding tele-education opportunities while travel is difficult or impossible. Achieving global health equity requires robust multilateral exchange; COVID-19 has demonstrated to us exactly how important the two-way street is.

We hope that better understanding the nature and value of GHEs will promote institutional support for global health education in North America and elsewhere. Valuable activities for supporting global health competencies include implementation of existing curricula (such as the tools published by the Consortium of Universities for Global Health ${ }^{22}$ or SUGARPREP $^{23}$ ); development of internal, specialty-specific curricula targeted at global health competencies; offering GHEs, whether in local communities or at partner sites elsewhere; and establishing global health tracks and opportunities to pursue advanced degrees while in clinical training (activities which also can support ACGME core competencies ${ }^{24}$ ). Delivering global health training requires hiring faculty with global health expertise and dedication of funds and faculty time to global health activities. It also requires putting in place administrative policies and staff to support trainees and faculty during GHEs. As GHEs typically involve partnership between high-resource institutions and vulnerable populations, it is also critical that global health programs be built with the input of both experts in global health and in the community where the GHE will take place.

We call for institutions to dedicate resources to global health because, as outlined here, we have recently seen how valuable global health education is to our institutions, our colleagues, and our learners. COVID-19 reminds us that everything global is also local. In times of disaster, shortage, and moral distress, local providers with global perspectives help health systems weather the storm.

Acknowledgements: The authors wish to acknowledge all of our partners working in chronically resource-challenged settings, without whose education, modeling, and guidance we would not have been able to as effectively respond to our own institutions' COVID-19 emergencies, nor gain the insights presented here.

Corresponding Author: Alexandra L. Coria, MD; Global Health Institute and Division of Pediatric Hospital Medicine, Maimonides Medical Center, Brooklyn, NY, USA (e-mail: ACoria@MaimonidesMed. org).

\section{Declarations:}

Conflict of Interest: Dr. Hudspeth and Dr. Rabin are co-chairs of the Education Committee of the Consortium of Universities for Global Health.

\section{REFERENCES}

1. Morens DM, Daszak P, Taubenberger JK. Escaping Pandora's Box Another Novel Coronavirus. New England Journal of Medicine. 2020;382 (14): 1293-1295. doi:https://doi.org/10.1056/nejmp2002106

2. Hooper MW, Nápoles AM, Pérez-Stable EJ. COVID-19 and Racial/ Ethnic Disparities. JAMA. Published online 2020. https://doi.org/10. 1001/jama.2020.8598

3. Kim EJ, Marrast L, Conigliaro J. COVID-19: Magnifying the Effect of Health Disparities. J Gen Intern Med. Published online May 11, 2020. https://doi.org/10.1007/s11606-020-05881-4

4. Koplan JP, Christopher Bond T, Merson MH, et al. Towards a common definition of global health. The Lancet. 2009;373(9679):1993-1995. https://doi.org/10.1016/s0140-6736(09)60332-9

5. Lu PM, Park EE, Rabin TL, et al. Impact of Global Health Electives on US Medical Residents: A Systematic Review. Ann Glob Health. 2018;84 (4):692-703

6. Aluri J, Moran D, Kironji AG, et al. The ethical experiences of trainees on short-term international trips: a systematic qualitative synthesis. BMC Med Educ. 2018;18(1):324.

7. Jeffrey J, Dumont RA, Kim GY, Kuo T. Effects of international health electives on medical student learning and career choice: results of a systematic literature review. Fam Med. 2011;43(1):21-28.

8. Nordhues HC, Usmaan Bashir M, Merry SP, Sawatsky AP. Graduate medical education competencies for international health electives: A qualitative study. Medical Teacher. 2017;39(11):1128-1137. doi:https:// doi.org/10.1080/0142159x.2017.1361518

9. Sawatsky AP, Nordhues HC, Merry SP, Bashir MU, Hafferty FW. Transformative Learning and Professional Identity Formation During International Health Electives: A Qualitative Study Using Grounded Theory. Acad Med. 2018;93(9):1381-1390.

10. Flatow V, Trinidad SM, Zhang LP, Marin ML, Divino CM. The Effect of a Global Surgery Resident Rotation on Physician Practices Following Residency: The Mount Sinai Experience. J Surg Educ. 2019;76(2):480486.

11. Lauden SM, Gladding S, Slusher T, Howard C, Pitt MB. Learning Abroad: Residents' Narratives of Clinical Experiences From a Global Health Elective. J Grad Med Educ. 2019;11(4 Suppl):91-99.

12. Russ CM, Tran T, Silverman M, Palfrey J. A Study of Global Health Elective Outcomes: A Pediatric Residency Experience. Glob Pediatr Health. 2017;4:2333794X16683806.

13. Harrison $\mathbf{J}, \operatorname{Logar} \mathbf{T}$, Le $\mathbf{P}$, Glass $\mathbf{M}$. What Are the Ethical Issues Facing Global-Health Trainees Working Overseas? A Multi-Professional 
Qualitative Study. Healthcare. 2016;4(3):43. doi:https://doi.org/10. 3390/healthcare4030043

14. Peluso MJ, Kallem S, Elansary M, Rabin TL. Ethical dilemmas during international clinical rotations in global health settings: Findings from a training and debriefing program. Med Teach. 2018;40(1):53-61.

15. Ogbasion H. Racism is a Global Health Issue. Published June 2, 2020 Accessed August 5, 2021. https://globalhealth.org/global-health-council-statement-on-recent-violence-against-black-americans /

16. Olusanya BO. Systemic racism in global health: a personal reflection. Lancet Glob Health. 2021;9(8):e1051-e1052.

17. Esquibel BM, O'Heron CT, Arnold EJ, Kallies KJ, Jarman BT. International Surgery Electives During General Surgery Residency: A 9 Year Experience at an Independent Academic Center. $J$ Surg Educ. 2018;75(6):e234-e239.

18. Balmer DF, Marton S, Gillespie SL, Schutze GE, Gill A. Reentry to Pediatric Residency After Global Health Experiences. Pediatrics. 2015; 136(4):680-686.

19. St Clair NE, Butteris SM, Cobb C, et al. S-PACK: A Modular and Modifiable, Comprehensive Predeparture Preparation Curriculum for Global Health Experiences. Acad Med. 2019;94(12):1916-1921.
20. Jacquet GA, Umoren RA, Hayward AS, et al. The Practitioner's Guide to Global Health: an interactive, online, open-access curriculum preparing medical learners for global health experiences. Med Educ Online. 2018;23 (1): 1503914 .

21. Marmot M, Friel S, Bell R, Houweling TAJ, Taylor S, Commission on Social Determinants of Health. Closing the gap in a generation: health equity through action on the social determinants of health. Lancet. 2008;372(9650): 1661-1669.

22. Global health competencies toolkit. Published April 21, 2020. Accessed March 25, 2021. https://www.cugh.org/online-tools/competenciestoolkit/

23. SUGARPREP. Accessed March 25, 2021. https://sugarprep.org/

24. Balasta MA, Khanal Y, McGinniss J, Moyer P, Rabin TL. Global Health Without Boundaries: Structuring Domestic and International Opportunities to Explore Global Health in a Graduate Internal Medicine Training Program. Ann Glob Health. 2016;82(6):1026-1033.

Publisher's Note: Springer Nature remains neutral with regard to jurisdictional claims in published maps and institutional affiliations. 